\title{
Salvage endoscopic ultrasound-guided rendezvous technique for disconnected pancreatic duct syndrome in a patient with severe acute pancreatitis
}

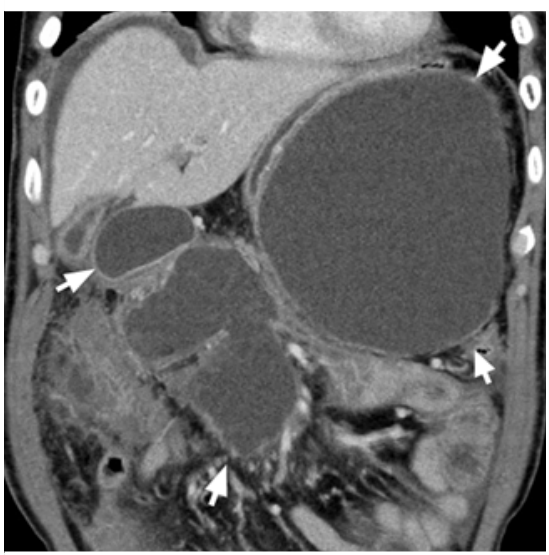

- Fig. 1 Computed tomography at the previous hospital revealed multiple walled-off necrosis (arrows).

Disconnected pancreatic duct syndrome (DPDS) is characterized by extraductal leakage of pancreatic juice and destruction of tissue surrounding the pancreas [1]. Many DPDS cases need surgical treatment [2]. Transpapillary pancreatic stenting and endoscopic ultrasound (EUS)-guided transmural drainage of PD and walled-off necrosis (WON) are also reported to be effective for DPDS [2 -4]. The EUS-guided rendezvous technique (EUS-RV) was shown to be effective as a salvage procedure to connect to the disruption directly when drainage procedures to treat DPDS proved ineffective.

A 60-year-old man suffered from severe pancreatitis after endoscopic retrograde cholangiopancreatography (ERCP) for PD stenosis of the pancreatic head. He was transferred to our hospital for further treatment because his WON-related symptoms (> Fig. 1) had worsened. We performed EUS-guided transmural drainage for the infected WON and percutaneous drainage for the abdominal effusion with a high amylase level. ERCP was performed for drainage to relieve the DPDS. Pancreatography showed only the proximal PD and extravasation of contrast medium in the WON ( $\triangleright$ Fig.2a). A nasocystic tube was placed in the WON
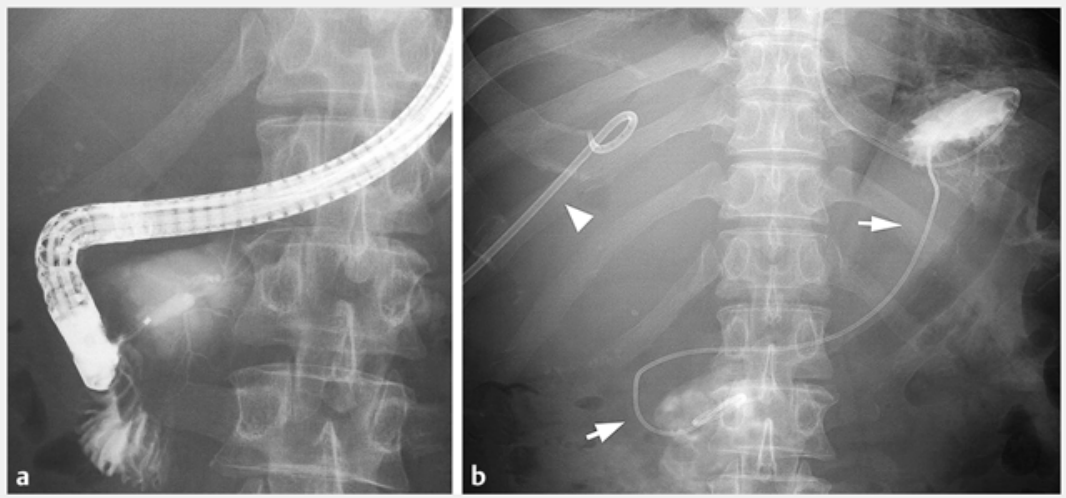

- Fig. 2 Pancreatography. a The proximal pancreatic duct without the distal duct and extravasation of contrast medium to the walled-off necrosis. b A nasocystic drainage tube (arrows) was placed in the walled-off necrosis that communicated with the pancreatic duct. Percutaneous drainage of the abdominal effusion had been performed previously (arrowhead).
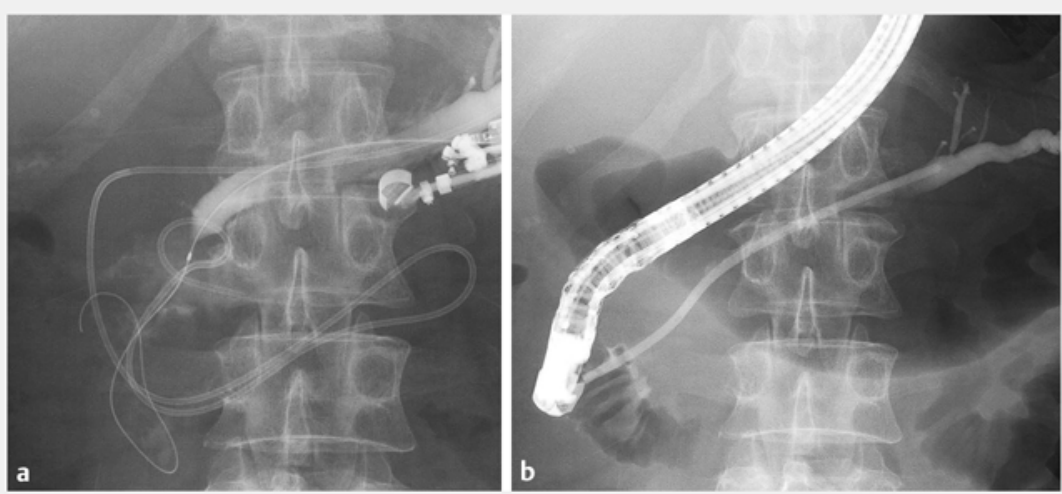

- Fig. 3 The endoscopic ultrasound-guided rendezvous technique. a A hydrophilic guidewire was advanced across the papilla of Vater after puncture of the pancreatic duct, using the nasocystic tube as a guide. b A pancreatic stent was placed to connect to the disconnected pancreatic duct.

via the PD because guidewire negotiation to the distal PD had failed ( $\triangleright$ Fig. 2b). Pancreatic juice still leaked, so EUSRV was performed to treat the DPDS ( Video 1).

The PD was punctured transgastrically by a 19-gauge needle (EZ shot 3 Plus; Olympus Medical, Tokyo, Japan), and a 0.025inch hydrophilic guidewire was manipulated through the duodenal papilla along the nasocystic tube ( $\mathbf{F i g} \cdot \mathbf{3 a}$ ). The echoendoscope was switched to a duodenoscope. The guidewire was grasped and brought into the accessory channel. Another catheter was cannulated over the guidewire to the PD. Finally, an 8.5$\mathrm{Fr}$ pancreatic stent (Olympus Medical) was placed across the disconnected PD ( Fig.3b). The exudate fluid was markedly reduced with external drainage, so 


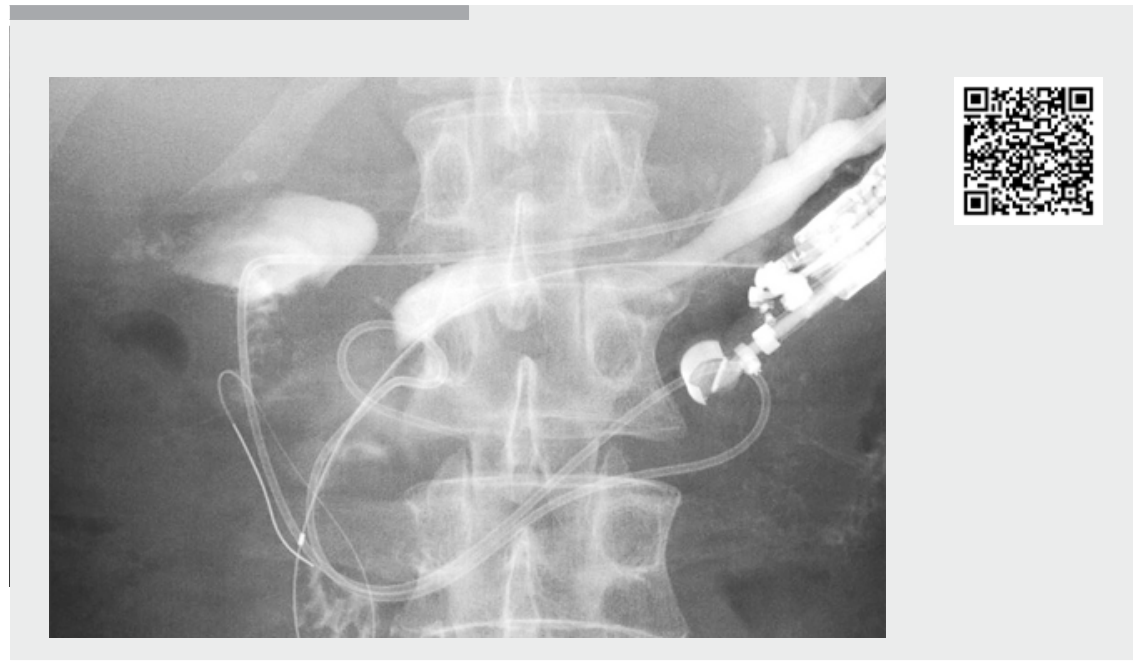

Video 1 Effective endoscopic ultrasound-guided rendezvous technique to connect to a pancreatic duct that had become disconnected due to severe acute pancreatitis.

the patient was transferred to the previous hospital 9 days after PD stenting without any complications.

Endoscopy_UCTN_Code_TTT_1AS_2AD

\section{Competing interests}

The authors declare that they have no conflicts of interest.

The authors

Shinichi Hashimoto, Hiromichi Iwaya, Shiroh Tanoue, Yusuke Fujino, Makoto Hinokuchi, Shiho Arima, Akio Ido

Digestive and Lifestyle Diseases, Kagoshima University Graduate School of Medical and Dental Sciences, Kagoshima, Kagoshima, Japan
Corresponding author

\section{Shinichi Hashimoto, MD}

Digestive and Lifestyle Diseases, Kagoshima University Graduate School of Medical and Dental Sciences, Kagoshima, 8-35-1 Sakuragaoka, Kagoshima 890-8520, Japan Fax: +81-99-2643504

kumdsh@m.kufm.kagoshima-u.ac.jp

\section{References}

[1] Pelaez-Luna M, Vege SS, Petersen BT et al. Disconnected pancreatic duct syndrome in severe acute pancreatitis: clinical and imaging characteristics and outcomes in a cohort of 31 cases. Gastrointest Endosc 2008 . 68: 91-97 connected pancreatic duct syndrome: endoscopic stent or surgeon's knife? Pancreas 2015; 44: 16-22
[2] Nadkarni NA, Kotwal V, Sarr MG et al. Dis-

[3] Rana SS, Bhasin DK, Sharma R et al. Factors determining recurrence of fluid collections following migration of intended long term transmural stents in patients with walled off pancreatic necrosis and disconnected pancreatic duct syndrome. Endosc Ultrasound 2015; 4: 208-212

[4] Krafft MR, Nasr JY. Anterograde endoscopic ultrasound-guided pancreatic duct drainage: a technical review. Dig Dis Sci 2019; 64 1770-1781

Bibliography

DOI https://doi.org/10.1055/a-1216-0809

published online 24.7.2020

Endoscopy 2021; 53: 450-451

Georg Thieme Verlag KG

Rüdigerstraße 14 ,

70469 Stuttgart, Germany

ISSN 0013-726X

\section{ENDOSCOPY E-VIDEOS \\ https://eref.thieme.de/e-videos}

口回 Endoscopy E-Videos is a free yccess online section, reporting 回: on interesting cases and new techniques in gastroenterological endoscopy. All papers include a high quality video and all contributions are freely accessible online.

This section has its own submission website at https://mc.manuscriptcentral.com/e-videos 